Cahiers de philosophie de l'université de

\title{
La défense esthétique du matérialisme diderotien
}

\section{Maud Pouradier}

\section{OpenEdition}

Journals

Édition électronique

URL : https://journals.openedition.org/cpuc/631

DOI : $10.4000 /$ cpuc.631

ISSN : 2677-6529

Éditeur

Presses universitaires de Caen

Édition imprimée

Date de publication : 31 décembre 2014

Pagination : $55-70$

ISBN : 978-2-84133-512-1

ISSN : 1282-6545

Référence électronique

Maud Pouradier, "La défense esthétique du matérialisme diderotien », Cahiers de philosophie de l'université de Caen [En ligne], 51 | 2014, mis en ligne le 13 juin 2018, consulté le 04 février 2023. URL http://journals.openedition.org/cpuc/631 ; DOI : https://doi.org/10.4000/cpuc.631

Creative Commons - Attribution - Pas d'Utilisation Commerciale 4.0 International - CC BY-NC 4.0 https://creativecommons.org/licenses/by-nc/4.0/ 


\title{
La défense esthétique du matérialisme diderotien
}

\begin{abstract}
Que non seulement l'image soit bien faite mais qu'elle soit aussi justifiée [...], et tout autant qu'une expérience cruciale, elle sera une contribution authentique à la connaissance.
\end{abstract}

Nelson Goodman ${ }^{1}$

\section{La raison des poètes}

Le principal argument de la Suite de l'entretien entre d'Alembert et Diderot en faveur du matérialisme est la continuité existant entre la matière inerte et la matière vivante: la manière dont la matière inerte devient vivante, en étant assimilée par l'animal dans le processus digestif, montre selon Diderot que la charge de la preuve revient aux partisans d'une âme spirituelle ayant pour fonction d'animer la matière inerte. La naissance de la conscience et de l'intelligence est éclairée par la métaphore du «clavecin sensible et animé». Dans un clavecin, un son fait résonner ses harmoniques. Que le clavecin soit doué de sensibilité et de mémoire, et alors il pensera et aura conscience de lui-même. Quel est le statut de cette comparaison? Peut-elle constituer un argument philosophique valide? Nous ne souhaitons pas ici étudier le matérialisme de Diderot en tant que tel, mais montrer comment les réflexions artistiques et esthétiques apportent des arguments en faveur de ce matérialisme.

Plusieurs éléments textuels nous y invitent. Dès la première page de l'Entretien entre d'Alembert et Diderot, Diderot tente de persuader d'Alembert en utilisant une image artistique et esthétique:

1. N. Goodman, Langages de l'art. Une approche de la théorie des symboles, J. Morizot (trad. fr.), Nîmes, J. Chambon, 1990, p. 56-57. 
D’Alembert : Je voudrais bien que vous me disiez quelle différence vous mettez entre l'homme et la statue, entre le marbre et la chair.

Diderot : Assez peu... On fait du marbre avec de la chair, et de la chair avec du marbre ${ }^{2}$.

Diderot ne se contente pas ici de préparer l'expérience de pensée du Falconet, broyé puis digéré par le moyen terme d'une plante comestible 3 . La possibilité de la représentation de l'une en l'autre semble montrer la continuité existant entre la matière dite inerte et la matière vivante. Le passage du représentant au représenté semble assis sur la possibilité réelle pour le marbre de devenir vivant. Ce n'est pas simple ironie de la part de Diderot.

Dans les Pensées sur l'interprétation de la nature, l'auteur établissait déjà une relation entre la loi de la nature et la loi de la représentation artistique: «Il me paraît aussi impossible que tous les êtres de la Nature aient été produits avec une matière parfaitement homogène, qu'il le serait de les représenter avec une seule et même couleur» ${ }^{4}$. L'art n'imite pas la nature telle quelle, contrairement à l'interprétation goethéenne de l'esthétique diderotienne ${ }^{5}$; mais la loi de l'art n'est pas seulement le signe de la loi de la nature ${ }^{6}$. La loi artistique et la loi naturelle sont dans une relation d'analogie, de sorte que l'impossibilité de représenter la nature avec une unique couleur montre qu'il doit exister plusieurs types de matière. C'est pourquoi la pure imitation du corps humain, sans la médiation de l'anatomie, conduit à la connaissance de la «liaison secrète» des phénomènes, sans pour autant que l'artiste pénètre pleinement les causes et les effets de la nature ${ }^{7}$. Mais l'Entretien va plus loin, suggérant que seul un représentant pouvant réellement devenir le représenté peut imiter avec succès ce qu'il prétend évoquer.

2. D. Diderot, Entretien entre d'Alembert et Diderot, in Euvres philosophiques, M. Delon (éd.), Paris, Gallimard (Bibliothèque de la Pléiade), 2010, p. 345.

3. Ibid., p. 347.

4. D. Diderot, Pensées sur l'interprétation de la nature, in Euvres philosophiques, p. 329. La réciproque est vraie: «Les productions de l'art seront communes, imparfaites et faibles, tant qu'on ne se proposera pas une imitation plus rigoureuse de la Nature»; ibid., p. 310. Il est vrai que dans cette dernière citation, Diderot évoque l'art en général, et non les seuls beaux-arts.

5. J. W. von Goethe, «L'Essai sur la peinture de Diderot», in Écrits sur l'art [1983], J.-M. Schaeffer (éd. et trad. fr.), Paris, Flammarion, 1996, p. 188 sq.

6. En ce sens pour Diderot, contrairement au Descartes de La Dioptrique, la représentation n'est pas assimilable à la signification.

7. D. Diderot, Essais sur la peinture, in Euvres, L. Versini (éd.), Paris, R. Laffont (Bouquins), t. IV, Esthétique-théâtre, 1996, p. 468. 
Diderot explique la possibilité de la sensation selon un raisonnement similaire: nous ne pourrions être sensibles à la matière inerte si la matière vivante n'était dans une continuité réelle avec la matière inerte - autrement dit si la matière inerte n’était elle-même sensible. Le processus sensitif («Nos sens sont autant de touches qui sont pincées par la nature $»^{8}$ ) est ainsi mis immédiatement en parallèle par Diderot avec la transformation de la matière «inerte» en matière «vivante»: «Qu'est-ce qu'un œuf? [...] D'abord c'est un point qui oscille, un filet qui s'étend et qui se colore, de la chair qui se forme, un bec, des bouts d'ailes, des yeux, des pattes, qui paraissent; une matière jaunâtre qui se dévide et produit des intestins; c'est un animal $» 9$. Le processus sensitif semble représenter le processus de vivification de la matière «inerte». Le premier suppose le second, et le second est vérifié par le premier. Dans l'Entretien, il ne s'agit cependant que d'un cercle adossé sur des métaphores. Comme le dit $\mathrm{M}^{\mathrm{lle}}$ de Lespinasse dans Le Rêve de d'Alembert: «les comparaisons sont presque toute la raison des femmes et des poètes ${ }^{10}$. Reste donc à fonder philosophiquement la raison des femmes et des poètes, pour faire de la métaphore un argument authentiquement philosophique. Ce qui pourrait rester un cercle est prouvé par l'évidence de l'affect esthétique, lieu de transformation de l'argument rhétorique en preuve.

\section{L'échec d'une esthétique matérialiste dans le «Traité du beau»}

À l'occasion de l'Encyclopédie quinze ans plus tôt, Diderot n'était pas parvenu à articuler pleinement esthétique et matérialisme. Le «Traité du beau » définit la beauté en termes de rapport ${ }^{11}$, par opposition à l'idée d'ordre, chère à la théologie naturelle et aux conceptions chrétiennes de la beauté ${ }^{12}$. En ce sens, on peut parler du «Traité du beau» comme d'une tentative de fonder une esthétique athée, et donc matérialiste ${ }^{13}$. La dernière page de cet essai est ici révélatrice:

8. D. Diderot, Entretien..., p. 352.

9. Ibid.

10. D. Diderot, Le Rêve de d'Alembert, in Euvres philosophiques, p. 369.

11. D. Diderot, Recherches philosophiques sur l'origine et la nature du beau, in Euvres, t. IV, p. $81 s q$.

12. «Saint Augustin avait composé un traité sur le beau, mais cet ouvrage est perdu, et il ne nous reste de saint Augustin sur cet objet important que quelques idées éparses dans ses écrits, par lesquelles on voit que ce rapport exact des parties d'un tout entre elles, qui le constitue UN, était, selon lui, le caractère distinctif de la beauté»; ibid., p. 82.

13. Il va sans dire qu'il ne s'agit nullement d'une esthétique matérialiste au sens adornien ou marxisant du terme. 
Le beau n'est pas toujours l'ouvrage d'une cause intelligente: le mouvement établit souvent, soit dans un être considéré solitairement, soit entre plusieurs êtres comparés entre eux, une multitude prodigieuse de rapports surprenants. Les cabinets d'histoire naturelle en offrent un grand nombre d'exemples. Les rapports sont alors des résultats de combinaisons fortuites, du moins par rapport à nous [...]; mais combien il faudrait remarquer de rapports dans un être pour avoir une certitude complète qu'il est l'ouvrage d'un artiste; en quelle occasion un seul défaut de symétrie prouverait plus que toute somme donnée de rapports; comment sont entre eux le temps de l'action de la cause fortuite, et les rapports observés dans les effets produits; et si, à l'exception des œuvres du Tout-Puissant, il y a des cas où le nombre des rapports ne puisse jamais être compensé par celui des jets ${ }^{14}$.

Définir le sentiment de la beauté comme la "perception de rapports ", c'est s'opposer à la définition de la beauté en termes d'ordre, opposer la multiplicité à l'unicité d'une supposée cause divine: «J'appelle donc beau hors de moi, tout ce qui contient en soi de quoi réveiller dans mon entendement l'idée de rapports; et beau par rapport à moi, tout ce qui réveille cette idée ${ }^{15}$. Le "Traité du beau» propose ainsi une conception dynamique de la beauté: il ne s'agit pas de déceler un ordre sous-jacent, mais de se montrer sensible à l'infinité des rapports suscités par le bel objet. En effet, Diderot propose une typologie complexe des rapports, insistant sur le fait qu'existent des « rapports fictifs» ayant pour origine la comparaison, la mémoire et l'imagination. Bien que ces rapports aient un fondement ontique dans l'objet sur lequel le jugement de goût est porté, ils dépendent donc des souvenirs et des expériences personnelles du spectateur. En outre, le jugement de goût a tendance à devenir électif, comme le montre l'opposition que fait Diderot entre le «beau absolu» et le «beau relatif»: le beau absolu est l'objet d'un jugement sur un être considéré comme un pur individu - ce qui éveille des rapports -, tandis que le beau relatif s'appuie sur une comparaison implicite avec les objets de la même espèce - telle fleur engendre plus de rapports que les autres en mon entendement. On comprend dans cette perspective qu'un même objet pourra être jugé beau d'un point de vue absolu et / ou relatif selon la connaissance et l'expérience du spectateur. Il est permis de supposer que plus le spectateur développe son goût et sa culture, et plus ses jugements esthétiques deviendront électifs - dynamique que l'on voit d'ailleurs à l'œuvre dans l'écriture même des Salons quelques années plus tard.

14. D. Diderot, Recherches philosophiques..., p. 112.

15. Ibid., p. 99 . 
Le concept de «rapports" - car c'est bien au pluriel que le pense Diderot - s'oppose à l'idée d'un ordre unitaire sous-jacent à la beauté. Leur multiplication non intentionnelle pourrait illustrer les harmoniques résonnantes du clavecin animé. Comme dans l'Entretien, Diderot use d'un argument prenant la forme d'un sorite: de même que petit à petit, dans le processus digestif, la matière inerte devient vivante - rendant impossible un critère de distinction net entre matière inerte et matière " animée»-, il est impossible de connaître le seuil à partir duquel le nombre de rapports prouve l'existence d'une cause unique et divine expliquant la beauté. À l'échelle du cosmos, c'est le "nombre de jets » qu'il faudrait comparer aux beaux objets: dans une veine épicurienne ${ }^{16}$, l'auteur interdit toute théologie naturelle fondée sur la beauté de la nature. Mais ce qui nous intéresse plus directement ici est la référence aux défauts de «symétrie»: dans le "Traité du beau », Diderot développe en effet l'importance des " dissonances », qui permettent, par contraste, de mettre en valeur les « rapports» engendrant le sentiment de la beauté. Loin de dissoudre la dissonance dans une résonance supérieure - argument typiquement théologique - Diderot souligne au contraire l'importance de la dissonance pour la dissonance, qui est certes mise en rapport avec les consonances, mais n'est pas pour autant «résolue » dans une consonance supérieure. Le concept de rapports permet ainsi de comprendre l'attrait du défaut dans une composition, sans passer par pertes et profits ce défaut, et tout en lui conservant son caractère défectueux. Il s'agit moins d'une esthétique de la résolution que d'une esthétique du contraste, cette dernière permettant une conception athée et matérialiste du beau.

Pourtant, Diderot abandonnera dans ses textes esthétiques ultérieurs, le vocable des «rapports». L'hypothèse d'une simple évolution des conceptions esthétiques de Diderot n'est pas satisfaisante. S'il est incontestable que la culture picturale et sculpturale de Diderot se déploie au cours de la rédaction des Salons, rendant ses écrits moins abstraits que le texte écrit à l'occasion de l'Encyclopédie, les thèses fondamentales de Diderot exposées dans le «Traité du beau» ne sont pas remises en cause dans les Salons et les textes qui leur sont contemporains. Comment expliquer la disparition du concept de "rapports " ? Il est incontestable que cette théorie déploie une conception intellectualiste de la beauté - d'où l'insistance de Diderot sur la fonction de l'entendement dans le jugement de goût. Toutefois, si les Essais sur la peinture et les Pensées détachées sur la peinture, la sculpture, l'architecture et la poésie insistent moins sur la fonction de l'entendement, Diderot ne développe jamais une conception strictement sensualiste de la beauté. Son

16. L'exergue des Pensées sur l'interprétation de la nature est une citation de Lucrèce. 
étude sur la composition et ses longs développements sur le dessin montrent assez que le beau demeure aussi perçu par l'entendement, et non simplement senti. Il semble toutefois que le concept de «rapports», que Diderot lie étroitement à l'entendement, maintient un dualisme «fonctionnel» dans le jugement esthétique entre un entendement purement intellectuel et une sensibilité réduite à n'apporter que la matière du jugement. Un tel dualisme «fonctionnel» semble plutôt un argument en faveur du dualisme réfuté dans l'Entretien, et si le jugement esthétique rend probable l'existence d'une substance spirituelle, les « rapports» pluriels risquent d'être subsumés sous l'unité d'un ordre théologique. Le dernier alinéa déjà cité du «Traité du beau " ne permet pas entièrement d'éloigner cette objection. Il semble au contraire souligner que la charge de la preuve incombe au matérialiste.

\section{Signification matérialiste de l'absorption}

C'est au contact des œuvres mêmes que l'esthétique de Diderot trouve son plein déploiement, et que l'affect esthétique du critique peut être le creuset d'une nouvelle argumentation en faveur du matérialisme. Un critère de jugement fréquent dans les Salons est la possibilité offerte par le tableau d'errer fictivement en son sein, sans pour autant entrer dans le dispositif scénique. Michael Fried a nommé «absorption» et "antithéâtralité» cette esthétique antirococo ${ }^{17}$. Au contraire, les toiles de Boucher ne cessent d'interpeller le spectateur et d'entrer dans son espace, quand le tableau de goût absorbe d'autant mieux le spectateur dans l'espace représenté qu'il ignore sa présence. Cette possibilité de l'absorption s'avère un argument en faveur du matérialisme. La critique du Rendez-vous de chasse du prince de Condé de Loutherbourg dans le Salon de 1765 est révélatrice.

Si quelqu'un ignore l'effet maussade de la symétrie, il n'a qu'à regarder ce tableau. Tirez une ligne verticale du haut en bas; pliez la toile sur cette ligne, et vous verrez la moitié de l'enceinte tomber sur l'autre moitié; à l'entrée de cette enceinte, un bout de barricade tomber sur un bout de barricade; en s'avançant de là peu à peu vers le fond, des chasseurs et des chiens tomber sur des chasseurs et des chiens; successivement une portion de la forêt tomber sur une égale portion de forêt; l'allée qui sépare ces deux portions touffues et la table placée au milieu de cette portion coupée en deux, tomber aussi, l'une des moitiés de la table sur l'autre moitié, l'une des moitiés de l'allée sur l'autre. Prenez des ciseaux et divisez par la ligne verticale la composition en deux lambeaux, et vous aurez deux demi-tableaux calqués l'un sur l'autre ${ }^{18}$.

17. M. Fried, La Place du spectateur [1980], C. Brunet (trad. fr.), Paris, Gallimard, 1990.

18. D. Diderot, Salon de 1765, in Euvres, t. IV, p. 400. 
Le problème du paysage de Loutherbourg est qu'il est tout en extériorité, au point qu'il semble s'engendrer lui-même par simples pliages et «étoilement ${ }^{19}$. C'est tout l'inverse des paysages où l'on vagabonde en pensée, happé tantôt derrière un bosquet, tantôt derrière une barrière ${ }^{20}$. Ce faisant, la construction de l'espace est comme en relief sur la toile (comme une "croûte de pain ${ }^{21}$ ). Le spectateur n'est ni entraîné vers la profondeur représentée de la toile, ni invité à se recueillir dans son intériorité. Celle-ci ne consiste certes pas à s'abîmer dans sa subjectivité ${ }^{22}$, mais à pouvoir entrer dans un espace fictif qui n'est pas l'espace dans lequel se meut mon corps. Or, pour un matérialiste rejetant la possibilité du cogito cartésien, qu'est-ce que la conscience ou l'intériorité sinon cet espace fictif dans lequel on peut s'abîmer, et permettant réellement d'oublier l'espace du corps? L'expérience esthétique est semblable à l'expérience de $\mathrm{M}^{1 \mathrm{le}} \mathrm{de}$ Lespinasse, découvrant, dans Le Rêve de d'Alembert, qu'elle est liée à l'univers entier:

J'existe [la nuit] comme en un point. Je cesse presque d'être matière. Je ne sens que ma pensée [...]. L'univers est anéanti pour moi; et je suis nulle pour lui $[\ldots]$. Il m'a semblé plusieurs fois en rêve [...] que mes bras et mes jambes s'allongeaient à l'infini, que le reste de mon corps prenait un volume proportionnée 23 .

Or, l'expérience esthétique, comprise comme une absorption, allie la contraction et la dilatation. Alors que l'expérience rythmique de $\mathrm{M}^{\text {lle }}$ de Lespinasse pourrait encore faire accroire que nous ne sommes pas seulement matière mais aussi âme immatérielle ( "Je cesse presque d'être matière. Je ne sens que ma pensée [...]»), l'expérience esthétique

19. G. Didi-Huberman, L'Étoilement. Conversation avec Hantaï, Paris, Minuit, 2013.

20. Ce critère n'est qu'apparemment contradictoire avec l'idée d'absorption. «Je dirais à présent que deux conceptions de l'art de peindre coexistent dans les Salons [... . La première d'entre elles, ou conception dramatique, demande qu'on établisse la fiction de l'inexistence du spectateur dans et par la représentation convaincante de personnages totalement absorbés dans leurs actions [...]. La seconde d'entre elles, ou conception pastorale, que l'on doit finalement voir comme un surgeon ou même une déclinaison particulière de la conception dramatique, demande qu'on établisse la fiction opposée, mais à bien des égards équivalente, d'une présence physique du spectateur dans le tableau. Il s'agit alors de recréer presque magiquement l'effet de la nature elle-même» ; M. Fried, La Place..., p. 131-132.

21. D. Diderot, Salon de 1765, p. 369.

22. Diderot rejette la possibilité de l'expérience cartésienne du cogito dans les «Additions à la Lettre sur les sourds et muets» : «Plusieurs fois, dans le dessein d'examiner ce qui se passait dans ma tête, et de prendre mon esprit sur le fait, je me suis jeté dans la méditation la plus profonde, me retirant en moi-même avec toute la contention dont je suis capable; mais ces efforts n'ont rien produit »; D. Diderot, "Additions à la Lettre sur les sourds et muets ", in Euvres philosophiques, p. 249.

23. D. Diderot, Le Rêve de d'Alembert, p. 382. Les références au stoïcisme sont évidentes dans Le Rêve de d'Alembert. Il n'est pas dans notre propos d'y insister. 
de l'absorption montre que la contraction ponctuelle est le revers d'une dilatation physique. C'est parce que je suis absorbé par l'espace représenté de la toile que je suis comme réduit à un point de l'espace réel. L'affect esthétique prouve ce que Le Rêve de d'Alembert ne faisait que suggérer ${ }^{24}$.

\section{Conspiration universelle et composition artistique}

Une telle expérience de contraction et de dilatation simultanées ne peut trouver son fondement que dans un monisme: si le dualisme était vrai, la concentration ne pourrait jamais se confondre avec la dilatation. «L'indépendance absolue d'un seul fait est incompatible avec l'idée de tout; et sans l'idée de tout, plus de philosophie ${ }^{25}$. Le premier fait indépendant à bannir est évidemment un principe spirituel sans commune mesure avec la matière. Diderot apporte des arguments artistiques en faveur de cette thèse.

Dans les Essais sur la peinture, Diderot insiste ainsi tout autant sur la nécessité de l'unité de la composition que sur celle de la variété, selon une «loi des énergies et des intérêts » que tente d'énoncer l'auteur dans le cinquième chapitre.

Si la scène est une, claire, simple et liée, j'en saisirai l'ensemble d'un coup d'œil; mais ce n'est pas assez. Il faut encore qu'elle soit variée [...]. Un homme fait une lecture intéressante à un autre. Sans qu'ils y pensent l'un et l'autre, le lecteur se disposera de la manière la plus commode pour lui; l'auditeur en fera autant $[\ldots]$. Ajoutez un troisième personnage à la scène, il subira la loi des deux premiers; c'est un système combiné de trois intérêts. Qu'il en survienne cent, deux cents, mille, la même loi s'observera [...]. Que l'artiste garde cette loi des énergies et des intérêts, et de quelque étendue que soit sa toile, sa composition sera vraie partout ${ }^{26}$.

$\mathrm{Au}$ contraire, les peintres académiques composent leurs personnages extérieurement, selon une loi des contrastes qui disperse les protagonistes et les construit mutuellement selon une logique superficielle ${ }^{27}$. L'idée de

24. «BORDEU: Voilà le dernier terme de la concentration de votre existence mais sa dilatation idéale peut être sans bornes. Lorsque la vraie limite de votre sensibilité est franchie, soit en vous rapprochant, en vous condensant en vous-même, soit en vous étendant au-dehors, on ne sait plus ce que cela peut devenir"; D. Diderot, Le Rêve de d'Alembert, p. 382. Bordeu suggère ici clairement que les deux expériences de $\mathrm{M}^{\text {lle }}$ de Lespinasse sont les revers d'un même phénomène.

25. D. Diderot, Pensées sur l'interprétation de la nature, p. 291.

26. D. Diderot, Essais sur la peinture, p. 497.

27. "Ce contraste d'étude, d'académie, d'école, de technique, est faux. Ce n'est plus une action qui se passe en nature, c'est une action apprêtée, compassée, qui se joue sur la toile»; ibid., p. 498 . 
«rapports» est ici approfondie et dépassée. La loi des énergies proposée par Diderot doit permettre de conserver la variété du naturel sans renier l'unité de la composition, et de peindre une action «en nature ${ }^{28}$, c'est-à-dire non seulement en conformité avec la nature, mais selon la loi même de la nature, selon laquelle il n'y a pas d'individus isolés mais seulement le tout.

Ne convenez-vous pas que tout tient en nature et qu'il est impossible qu'il y ait un vide dans la chaîne? Que voulez-vous donc dire avec vos individus? Il n'y en a point. Non, il n'y en a point... Il n'y a qu'un seul grand individu; c'est le tout ${ }^{29}$.

Cette loi des énergies est l'équivalent artistique des forces d'attraction des Pensées sur l'interprétation de la nature ${ }^{30}$. Dans Le Rêve de d'Alembert, la remise en cause du concept d'individu est un argument en faveur de la continuité entre la matière inerte et la matière vivante: l'individu est assimilé par Diderot à un saut, que la nature ne souffre pas. Sa remise en cause va de pair avec celle d'une âme supposée fonder cette individualité. L'esthétique tout en extériorité de l'académisme rococo repose sur l'hypothèse d'individus isolés, rapprochés arbitrairement par un principe extérieur (Dieu ou peintre). Une représentation construite selon un tel principe n'est pas «vraie», non au sens où le représentant devrait se confondre avec le représenté - l'imitation parfaite n'est qu' «analogue aux causes ${ }^{31}-$, mais au sens où elle n'est pas réussie en tant que représentation. Cet échec est signé par un affect esthétique négatif, la perception esthétique ayant été élevée au rang de critère de jugement de la vérité de l'imitation dans les premières pages des Essais sur la peinture ${ }^{32}$. La composition rêvée par Diderot doit au contraire nous persuader de la primauté du tout sur l'individu, et d'une conception moniste de l'univers.

\section{L'air et le vide}

La primauté du tout sur l'individu, et la réalité de la continuité physique, trouvent une autre expression dans l'esthétique «atmosphérique» de Diderot, par opposition à l'esthétique rococo $\mathrm{du}$ « vide». Citons par exemple sa critique d'Andromaque éplorée devant Ulysse de Doyen en 1763:

28. Ibid. On trouve également cette expression dans Le Rêve de d'Alembert, p. 371.

29. D. Diderot, Le Rêve de d'Alembert, p. 371.

30. Pensée XXXVI.

31. D. Diderot, Essais sur la peinture, p. 468.

32. «Plus l'imitation serait parfaite et analogue aux causes, plus nous en serions satisfaits »; ibid. 
Et ce vide énorme qui sépare Ulysse de la scène, et qui le relègue à une distance choquante? Il coupe la composition en deux parties dont on ferait deux tableaux distincts, l'une à conserver précieusement, l'autre à jeter au feu ${ }^{33}$.

Ce vide - tant spatial qu'affectif - brise l'unité compositionnelle. De même, c'est le vide entre les membres d'un corps nu ${ }^{34}$ qui le transforme en corps "mannequiné », celui entre les objets d'une nature morte qui les transforme en "accessoires ${ }^{35}$. Au contraire, le talent de Chardin consiste à montrer l'humidité et les couleurs diaphanes de l' "air». Le style, «c'est l'art de rendre cet air pour ainsi dire sensible, cette vapeur légère qui règne dans les grands édifices ${ }^{36}$. Si l'on en croit la critique de Boucher dans le Salon de 1765, l'esthétique du vide est une esthétique de la séparation, de la décomposition, qui transforme le tableau en pure extériorité (c'est la "croûte de pain» des toiles académiques, l' «anatomie » des nus d'école). C'est une esthétique du détail accessoire mis en avant de manière extraordinaire. Telle est l'obscénité fondamentale de Boucher. Une telle esthétique suppose que le réel est constitué d'individus. Son échec à représenter la nature, tant d'un point de vue artistique qu'esthétique, montre a contrario que la nature n'est pas constituée d'individus isolés les uns des autres par un vide causal. Diderot ne rejette pas la possibilité du vide en tant que tel, mais la possibilité que deux êtres soient sans influence l'un sur l'autre. En ce sens, Diderot se fait le défenseur d'une physique newtonienne, comme le montre son insistance sur les concepts de force et d'attraction dans les Pensées sur l'interprétation de la nature.

L'esthétique "atmosphérique» d'un Chardin souligne au contraire la manière dont la vie est capable d'irradier autour du corps, dont les objets sont liés et s'influencent à distance réciproquement. Telle semble être la signification de l' «air» dans la peinture, par opposition au "vide». Corrélativement, la représentation souligne la manière dont la vie transpire de la matérialité elle-même, comme le montre la célèbre description de La Raie dépouillée de Chardin.

Ce sont des couches épaisses de couleur, appliquées les unes sur les autres, et dont l'effet transpire de dessous en dessus. D'autres fois on dirait que c'est une vapeur qu'on a soufflée sur la toile; ailleurs, une écume légère qu'on y a jetée. [...] Approchez-vous, tout se brouille, s'aplatit et disparaît. Éloignez-vous, tout se recrée et se reproduit ${ }^{37}$.

33. D. Diderot, Salon de 1763, in Euvres, t. IV, p. 282.

34. On peut lire sous cet angle la critique de Boucher dans le Salon de 1765, p. 312 sq.

35. Ibid., p. 359-36o.

36. D. Diderot, Salon de 1763, p. 281. Également: Salon de 1765, p. 345.

37. D. Diderot, Salon de 1763, p. 265. 
Le miracle de la représentation renvoie au miracle même du corps animal - c'est pourquoi les natures mortes de Chardin paraissent mystérieusement vivantes, et peuvent être mises esthétiquement sur le même plan que le grand genre représentant la réalité humaine ${ }^{38}$. Dans cette description du phénomène de la représentation, Diderot souligne que le fonctionnement de la représentation est le fonctionnement même de la matière: elle est morte de près et vivante de loin, comme la représentation picturale n'est qu'aplats de peinture de près et vie même de loin. Ce n'est pas le spectateur qui observe et calcule ce que les aplats de couleur représentent. La matière picturale ellemême «se brouille» puis "se recrée». L'émergence de la représentation mime l'émergence de la vie à partir de l'inerte. Mais la description de l'expérience esthétique de la réussite artistique permet de sortir du cercle rhétorique de l'Entretien. Le spectacle de La Raie dépouillée montre que la représentation n'est pas une signification à déchiffrer, mais se constitue d'elle-même dans la matière. Le fait de la représentation artistique appuie la thèse matérialiste. Le lien entre représentant et représenté est plus que métaphorique.

\section{Lazare et Galatée}

Mais c'est évidemment la représentation même de la transformation de la matière inerte en matière vivante qui peut être le creuset d'arguments artistiques et esthétiques en faveur du matérialisme diderotien. La comparaison de la critique de La Résurrection de Lazare de Deshays et du Pygmalion au pied de sa statue qui s'anime de Falconet dans le Salon de 1763 est instructive ${ }^{39}$. Pour Diderot, le tableau de Deshays est manqué, quand la sculpture de Falconet est un chef-d'œuvre. Or l'auteur des Salons commence par faire remarquer que les erreurs du peintre ne sont pas ici seules en cause: «Dites-moi aussi pourquoi tous les ressuscités sont hideux. Il me semble qu'il vaudrait autant ne pas faire les choses à demi, et qu'il n'en coûterait pas plus de rendre la santé avec la vie ${ }^{40}$. Dans le Salon de 1759, Diderot s'était également montré très sévère envers La Résurrection de Jésus-Christ de Bachelier: «Ce Christ est tout disloqué. C'est un patient dont les membres ont été mal reboutés ${ }^{41}$. Quatre ans plus tard, tout se passe comme si le thème même de la résurrection était désormais critiqué. En effet, le tableau

38. C. Talon-Hugon, «Diderot et la question de la critique éthique de la peinture», in Diderot, l'expérience de l'art: "Salons de 1759, 1761, 1763" et "Essais sur la peinture", G. Cammagre et C. Talon-Hugon (dir.), Paris - Poitiers, PUF - CNED, 2007, p. 66-85.

39. D. Diderot, Salon de 1763, p. 262 et p. 286.

40. Ibid., p. 261.

41. D. Diderot, Salon de 1759, in CEuvres, t. IV, p. 198. S. Lojkine, L'Eil révolté. Les "Salons" de Diderot, Paris - Arles, J. Chambon - Actes Sud, 2007, p. 136-142. 
de Deshays est loin de manquer de qualités. Il n'est pas le fait d'un ridicule petit maître comme Bachelier. "Sans plaisanter, ce morceau n'est pas sans effet. Les groupes en sont bien distribués. Le Lazare avec son linceul est peint largement ${ }^{42}$. Si Diderot lui oppose le chef-d'œuvre de Jouvenet, c'est moins pour louer le traitement du thème biblique en tant que tel que pour la force expressive de Lazare et des spectateurs de la scène ("Quelle force d'expression! Quelle joie! Quelle reconnaissance!»), de sorte que la «vie» dévoilée par Jouvenet est moins celle du ressuscité que la vivacité de l'affect de Lazare et le naturel de sa représentation ${ }^{43}$. En tant que scène de résurrection, le chef-d'œuvre de Jouvenet est aussi manqué que le tableau de Deshays. «Mais que penseriez-vous de moi, si j'osais vous dire que toutes ces têtes de ressuscités, belles sans doute et du plus grand effet, sont fausses? ${ }^{44}$. Si les tableaux de Deshays et Jouvenet sont manqués, c'est parce que Lazare ressuscitant est conscient qu'il ressuscite - alors qu'il devrait l'ignorer et se contenter de jouir de ses sensations. Lazare ne peut donc en aucune façon être reconnaissant envers son sauveur, puisqu'il doit ne pas comprendre ce qui lui arrive, et croire tout au plus qu'il a perdu connaissance. Les peintres auraient donc dû se contenter de représenter la grâce de l'éveil, «celle d'un homme qui sort d'un profond sommeil ou d'une longue défaillance» ${ }^{45}$.

Cette erreur commune de Deshays et Jouvenet n'est toutefois pas entièrement de leur fait: c'est la scène même de l'Évangile de Jean qui est en cause. La seule résurrection vraiment sublime est celle peinte par Rembrandt, qui cache le visage de Lazare ${ }^{46}$. Pourquoi l'éveil de Lazare ou du Christ serait-il impossible à peindre en tant que tel, quand l'éveil de Galatée est possible sous les ciseaux de Falconet dans Pygmalion?

Ses yeux viennent de s'entrouvrir. Sa tête est un peu inclinée vers la terre, ou plutôt vers Pygmalion qui est à ses pieds. La vie se décèle en elle par un souris léger qui effleure sa lèvre supérieure. Quelle innocence elle a! Elle en est à sa première pensée. Son cœur commence à s'émouvoir; mais il ne tardera pas à lui palpiter. Quelles mains! Quelle mollesse de chair ${ }^{47}$ !

42. D. Diderot, Salon de 1763, p. 261.

43. D. Diderot, Essais sur la peinture, p. 486.

44. D. Diderot, Salon de 1763, p. 261.

45. Ibid., p. 262.

46. "Son Christ a l'air d'un tristo, il est à genoux sur le bord du sépulcre, il prie, et l'on voit s'élever deux bras du fond du sépulcre»; D. Diderot, Essais sur la peinture, p. 484. Cette description ne correspond à aucune version connue de Rembrandt. Diderot semble ici avoir fait la synthèse de deux versions différentes. Diderot ne voulait-il pas, dans le Salon de 1763 , proposer lui-même une composition du thème biblique à un peintre?

47. D. Diderot, Salon de 1763, p. 286. 
Il est surprenant que la sculpture soit plus adéquate que la peinture à la représentation de l'éveil de la matière morte, car «c'est le dessin qui donne la forme aux êtres; c'est la couleur qui leur donne la vie ${ }^{48}$. Sans doute l'absence de couleur du groupe sculpté renforce-t-elle la continuité entre la matière inerte du marbre et le corps vivant de Pygmalion, dont Galatée serait le moyen terme. Il semble cependant que le thème littéraire de Pygmalion soit plus propice que le thème biblique: l'objet du récit semble ici plus en cause que le medium.

L'éveil de Galatée symbolise le miracle de la représentation de la mollesse de la chair dans la dureté minérale. Galatée s'éveille peu à peu - elle «commence à s'émouvoir » et bientôt sa chair palpitera - comme la pierre imite progressivement la douce chair féminine. Le regard de Pygmalion symbolise son travail de sculpteur: ses yeux animent la pierre comme le font ses ciseaux. Est alors mise en abyme la contemplation même du spectateur, qui passe de la conscience de la pierre à la vision hallucinatoire d'une chair. «Appuyez-y votre doigt, et la matière qui a perdu sa dureté, cédera à votre impression ${ }^{49}$. Tandis que le geste de Pygmalion devient regard dans le groupe sculpté, dans le discours critique, le regard du spectateur devient geste. Dans ce chiasme réside l'étirement temporel de l'œuvre de Falconet, la durée esthétique du spectacle étant équivalente à la durée d'un cisèlement, lequel est allégoriquement l'éveil progressif du personnage féminin.

$\mathrm{Au}$ contraire, la difficulté du thème de la résurrection réside dans le fait qu'elle a lieu en un instant, celui du «Lazare lève-toi!» ordonné par le Christ. La représentation de la résurrection devrait donc mieux convenir à la peinture que l'éveil de Galatée, puisque la peinture est réduite à l'instant, contrairement à la poésie. «Le peintre n'a qu'un instant, et il ne lui est pas plus permis d'embrasser deux instants que deux actions $"{ }^{50}$. Mais la résurrection s'avère un instant irreprésentable, non pour des raisons théologiques ( Galimatias métaphysico-théologique ${ }^{51}$ ), mais proprement artistiques. Il est impossible de dépeindre le soudain passage de la mort à la vie. Ceci n'est pas propre à la résurrection, mais à tout événement dans l'instant de son surgissement. Les peintres ont depuis longtemps trouvé un moyen de représenter le moment d'une catastrophe. "Une catastrophe subite surprend un homme au milieu de ses fonctions; il est à la catastrophe, et il est encore à ses fonctions ${ }^{52}$. Mais il est impossible de

48. D. Diderot, Essais sur la peinture, p. 472.

49. D. Diderot, Salon de 1763, p. 286.

50. D. Diderot, Essais sur la peinture, p. 496.

51. D. Diderot, Entretien..., p. 353.

52. D. Diderot, Essais sur la peinture, p. 496-497. 
représenter Lazare encore mort et déjà vivant, sauf à transformer la scène de la résurrection en une animation progressive sur le modèle de Galatée.

Il faut donc se résoudre à évoquer la résurrection en représentant l'instant qui la précède, ou l'instant qui la suit. Représenter l'instant précédent n'aurait pas d'intérêt. Il faut donc représenter l'instant suivant la résurrection. Pour évoquer indirectement l'instant irreprésentable de la résurrection, le peintre doit attribuer à Lazare la conscience de sa propre résurrection, bien qu'elle soit incongrue. Toute résurrection est donc rigoureusement irreprésentable. Une telle tentative ne peut qu'aboutir à un échec artistique et esthétique - tous les ressuscités sont hideux malgré leurs belles têtes et leurs belles expressions. Le tableau de Rembrandt n'est qu'apparemment une exception à cette règle, car le maître a choisi judicieusement de ne représenter ni le visage de Lazare ni le «Lève-toi!» du Christ. Bien qu'indirecte, c'est la seule solution au problème artistique de la résurrection. «[I]l prie, et l'on voit s'élever deux bras du fond du sépulcre ${ }^{53}$. Ce faisant, la résurrection de Lazare est traitée comme l'animation de Galatée, la prière du Christ étant ici l'équivalent du regard de Pygmalion. L'idée sublime de Rembrandt de cacher le visage de Lazare pour ne laisser qu'entrapercevoir ses bras sortant du tombeau, permet d'évoquer l'instant irreprésentable de la résurrection, sans tomber dans la fiction d'un personnage ayant conscience d'avoir été mort, et sans transformer effectivement l'instant de la résurrection en un éveil progressif.

Le génie de Rembrandt ne nous fera cependant pas croire en la résurrection. À propos d'une méchante Résurrection de Jésus-Christ de Briard, Diderot écrit dans le Salon de 1765: "Comme cela est fait! Miséricorde! Ce Christ est si mince, si fluet, qu'il ferait douter de la Résurrection, si l'on y croyait, et croire à la palingénésie, si l'on en doutait » ${ }^{54}$. Point de boutade ici. La palingénésie dont il est question est moins celle de Chrysippe que celle de l'Entretien entre d'Alembert et Diderot et du Rêve de d'Alembert, et désigne plutôt la perpétuelle transformation du mort en vivant et réciproquement ${ }^{55}$. Au-delà du médiocre talent de Briard, l'impossibilité artistique de représenter la résurrection la rend impossible à croire. Non pas qu'un corps mort ne puisse revivre. Ce qui n'est pas crédible dans la résurrection et que les œuvres d'art mettent en lumière dans leur réussite biaisée - comme chez

53. D. Diderot, Essais sur la peinture, p. 484.

54. D. Diderot, Salon de 1765 , p. 394.

55. La version diderotienne de la palingénésie serait peut-être exprimée par d'Alembert dans Le Rêve de d'Alembert: "Tout change. Tout passe. Il n'y a que le Tout qui reste. Le monde commence et finit sans cesse. Il est à chaque instant à son commencement et à sa fin. Il n'en a jamais eu d'autre et il n'en aura jamais d'autre »; D. Diderot, Le Rêve de d'Alembert, p. 365 . 
Rembrandt - ou dans leur échec - comme chez Deshays ou Jouvenet -, c'est la possibilité d'un passage brutal de la mort à la vie. Un passage progressif s'avère en revanche représentable - comme le montre l'éveil de Galatée selon Falconet - et donc crédible; en ce sens, la réussite de Falconet est un argument artistique en faveur du monisme de Diderot.

Le matérialisme de Diderot s'appuie sur des arguments rhétoriques et esthétiques (métaphore du clavecin animé dans l'Entretien, de l'araignée au milieu de sa toile dans Le Rêve). La raison des poètes devient raison philosophique si l'on prend acte de la conception diderotienne de la représentation. Contre la définition cartésienne de la représentation en termes de signification, Diderot soutient non seulement que la loi de la représentation est analogue à la loi de la nature, mais qu'il y a ressemblance véritable entre représentant et représenté. Les moyens de la représentation donnent à voir la manière dont la nature procède, et la réussite de la représentation révèle la continuité entre un représentant inerte et un représenté vivant, voire pensant. Ce postulat devient axiome dès lors qu'il est assis par l'affect esthétique, faisant ici fonction d'évidence. L'expérience esthétique légitime ainsi en retour la raison des poètes, et l'usage philosophique des métaphores pour prouver le matérialisme.

Maud Pouradier

Université de Caen Basse-Normandie Identité et Subjectivité (EA 21 / 29) 